\title{
Mental Fatigue Measurement Based on the Changes in Flicker Perception Threshold using Consumer Mobile Devices
}

\author{
Sunao IwAKI ${ }^{*}$, , Nobuyoshi HARAdA ${ }^{* *}$
}

\begin{abstract}
We developed a technique to evaluate mental fatigue quantitatively by measuring perceptual performance of visually presented flicker; namely, the flicker-perception threshold (FPT), on consumer devices. Frequency domain flicker-perception threshold (FD-FPT) or critical fusion frequency (CFF), defined as a frequency at which intermittency of light disappears into a steady state for a human observer, is known to decrease as mental fatigue increases. Although FD-FPT has a long history as a reliable index of mental fatigue in the laboratory setting, it requires a dedicated device to measure, which impedes the use of this method by the general public. Additionally, arbitrariness and subjectivity of subject responses in the conventional flicker test present a significant problem for robust measurements of mental fatigue in everyday situations. This issue is especially important under such conditions where there is no supervision for users on how to respond to the ongoing flicker stimuli. We developed (a) a new method to control the subjective flickering sensation by changing the contrast of the visual stimuli instead of changing the temporal frequency, and ( $b$ ) a new paradigm in which forced-choice task performance is used to determine FPT to eliminate arbitrariness of subject responses. The proposed techniques were implemented as application software, which enables persons to measure mental fatigue quantitatively using consumer mobile devices such as smart phones and PCs.
\end{abstract}

Keywords : mental fatigue, flicker perception, personal mobile device, contrast threshold.

Adv Biomed Eng. 2: pp. 137-142, 2013.

\section{Introduction}

Frequency domain flicker-perception threshold (FDFPT) or critical flicker frequency $(\mathrm{CFF})$ is defined as a frequency at which intermittency of light disappears into a steady state for a human observer. The CFF is known to decrease as mental fatigue increases [1]. Although the CFF has been used for decades as a robust index of mental fatigue (the flicker test) in laboratory settings, especially in the field of ergonomics and occupational health [2], it has not been widely used by the general public. This is primarily because the test requires a dedicated device and measurement by a trained professional. Also, arbitrariness and subjectivity in subject responses during a conventional flicker test present significant problems for robust measurements of mental fatigue in everyday situations. Mainly, this is due to the absence of an experimenter or supervisor to instruct the users about their responses to ongoing flicker stimuli.

This study was presented at the Symposium on Biomedical Engineering 2013, Fukuoka, September, 2013.

Received on July 26, 2013; revised on October 2, 2013 and October 18, 2013; accepted on October 31, 2013.

* Human Technology Research Institute, National Institute of Advanced Industrial Science and Technology (AIST), Tsukuba, Japan.

** Flicker Health Management Corp., Ikeda, Japan.

\# AIST Tsukuba Central 6, 1-1-1 Higashi, Tsukuba, Ibaraki 305-8566, Japan.

E-mail: s.iwaki@aist.go.jp
The primary aim of this research was to develop a system to evaluate mental fatigue quantitatively and objectively, by measuring the flicker-perception threshold (FPT) on consumer devices such as cell-phones and PCs. Users of these devices do not have precise control of the temporal frequency of visual stimuli due to fixed display refresh rates. The main topics of the present study were:

(i) To develop and validate visual stimuli capable of gradually changing the magnitude of subjective flickering sensation on raster-scan display devices with fixed frame rates;

(ii) To design a psychophysical procedure capable of solving the inherent long-standing problems in the original flicker test; namely, arbitrariness and subjectivity in subject responses during FPT determination, and

(iii) To construct a system that can store and retrieve measured data online.

\section{Methods}

\subsection{Contrast-Controlled Flicker Stimuli (CCFS)}

To assess mental fatigue by measuring CFF (a frequencydomain FPT), a flickering light generated by an LED has been the primary method for manipulating temporal frequency of the flicker in resolutions under $1 \mathrm{~Hz}$. One of the main goals of this study was to develop a method to measure FPT on consumer devices. However, visual displays on these devices have fixed frame rates of typically 30 or 60 frames per seconds (fps), and it is impossible to change the frequency of the flickering stimuli to a resolution sufficient for $\mathrm{CFF}$ measurement.

In this study, instead of changing the temporal 
frequency as in the original flicker test, we developed a method to control the magnitude of subjective flickering sensation by changing the contrast between the on and off periods of the flickering visual stimuli (contrastcontrolled flicker stimuli; CCFS). This can be implemented on display devices with fixed frame rates. The concept behind CCFS is based on reciprocity between $\mathrm{CFF}$ and the luminance of the visual stimuli (i.e. brightness contrast of the flickering target stimuli against background) $[3,4]$.

To validate the compatibility of the proposed method (CCFS test) with the original flicker test, we conducted fatigue measurement tests on 12 subjects (all males, $32.9 \pm 10.8$ years of age). All subjects were non-smokers, were in good physical health and taking no medication, and had no reported history of psychiatric or somatic disorders. The experimental protocols were approved by the internal review board of National Institute of Advanced Industrial Science and Technology (AIST). Written informed consent was obtained from each subject prior to the experiment.

Subjects were required to continue their office work, typically word processing on PCs, overnight from $2: 30$ p.m. to $8: 30$ am the next morning. From $9: 00$ a.m. to $12: 00$ noon on the second day, the subjects took naps of up to 3 hours in individual rooms. Some subjects performed physical exercises such as swimming during the first night as part of their daily routine. Evaluation of changes in mental fatigue over this time period for each subject began at 2:30 p.m. on the first day of the experiment, and the subjects were subsequently evaluated every two hours. These evaluations consisted of (a) the CCFS flicker test implemented on a standard PC with a simulated fixed frame rate of $30 \mathrm{fps}$ (1 degree visual angle, viewed from a distance of $400 \mathrm{~mm}, 150 \mathrm{~cd} / \mathrm{m}^{2}$ for high brightness stimuli and $4.2 \mathrm{~cd} / \mathrm{m}^{2}$ for the lowest brightness stimulus), ( $b$ ) the CCFS flicker test implemented as a cell-phone application with a fixed frame rate of $15 \mathrm{fps}$ (1 degree visual angle, viewed from a distance of $300 \mathrm{~mm}, 70 \mathrm{~cd} / \mathrm{m}^{2}$ for the high brightness stimulus and $2.5 \mathrm{~cd} / \mathrm{m}^{2}$ for the lowest brightness stimulus), and (c) the original flicker test (Roken Digital Flicker Model RDF-1; Sibata Scientific Technologies Ltd). The experiment was carried out in normal office ambient light condition. Subjective rating of mental fatigue by visual analog scale (VAS) [5] was also collected immediately after each FPT measurement.

\subsection{Forced-Choice Task for FPT Detection (FC-FPT)}

The original flicker test uses FPT measurements based on voluntary responses from the subjects regarding the subjective sensation of the flicker. The arbitrariness of subject responses may introduce random biases on the fatigue measurements that could reflect subjects' intentions to manipulate results. This can pose major problems especially if the proposed technique is used to measure fatigue in everyday situations without ensuring proper training and/or supervision.

To address this problem, we designed a forced choice task for FPT detection (FC-FPT) in which subjects are required to choose a CCFS target among other nonflickering stimuli (Fig. 1).

The contrast of the CCFS target, which is defined as a difference in luminance of the flickering stimulus between $\mathrm{ON}$ (brighter) and $\mathrm{OFF}$ (darker) periods, decreased if the subject responded correctly, and the contrast increased if the subject's response was incorrect. The FC-FPT trials were repeated until the target contrast reached convergence.

\section{Results}

Figure 2 shows the results of fatigue measurements for a typical subject. Normalized (to initial measurement) FPT for the three methods of measurement: (a ) CCFS on PC, ( b ) CCFS on cell phone, and ( c ) original flicker test, are depicted. Results obtained using the CCFS tests ( $\mathrm{a}$ and $\mathrm{b}$ ) showed temporal characteristics similar to that obtained using the original flicker test ( $\mathrm{c}$ ). With all three methods of measurement, normalized FPT obtained after a nap between $9: 00 \mathrm{a} . \mathrm{m}$. and 12:00 noon returned to that close to the initial level in all subjects, representing recovery from mental fatigue imposed by overnight workload. Normalized FPT scores also increased temporarily after physical exercise for all three methods of measurement.

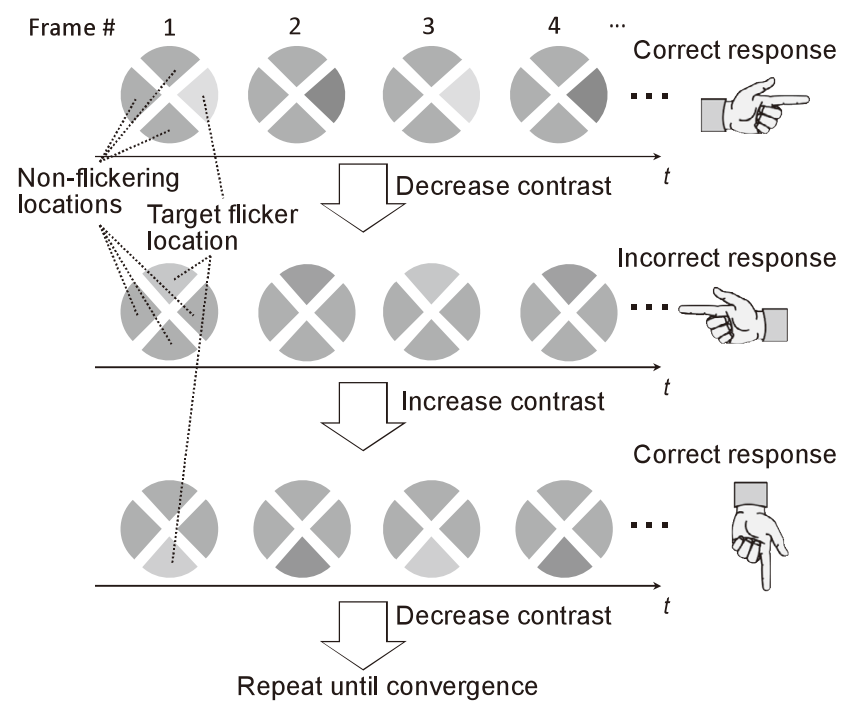

Fig. 1 Schematic representation of FPT detection using forced-choice task (FC-FPT). In the current implementation of FC-FPT, four wedges were presented on the display, one of which changed its luminance at every frame (target flicker) while the others stayed static. Subject's task was to choose the flickering location among the other non-target locations. Locations of the target stimuli were changed randomly in each trial. The contrast, which is a difference in target luminance between $\mathrm{ON}$ and $\mathrm{OFF}$ periods, decreased if the subject's response was correct, and the contrast increased if the subject responded incorrectly. The trials were repeated until the contrast converged to perception threshold. 


\section{Normalized FPT}

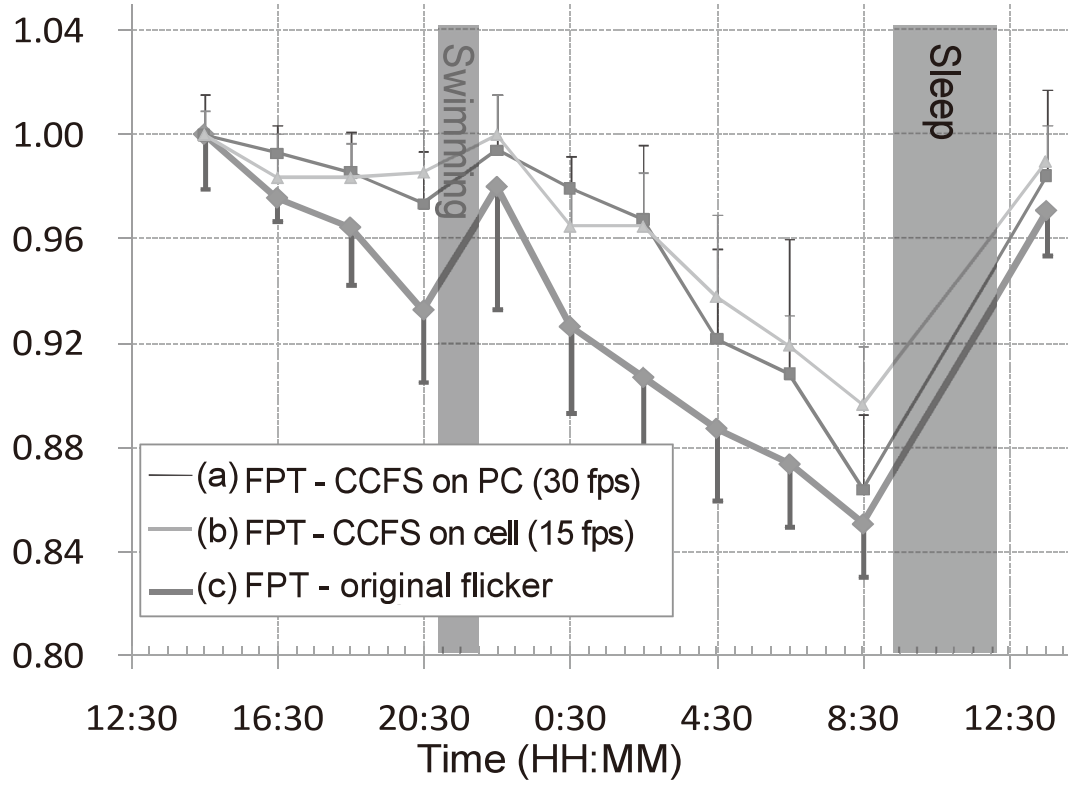

Fig. 2 Results of FPT measurements (mean \pm SD) during overnight office work setting in a typical subject using (a ) CCFS implemented as PC software, ( b ) CCFS implemented on standard cell-phone application, and ( $\mathrm{c}$ ) the original flicker test. FPT was measured 5 times at each time point.

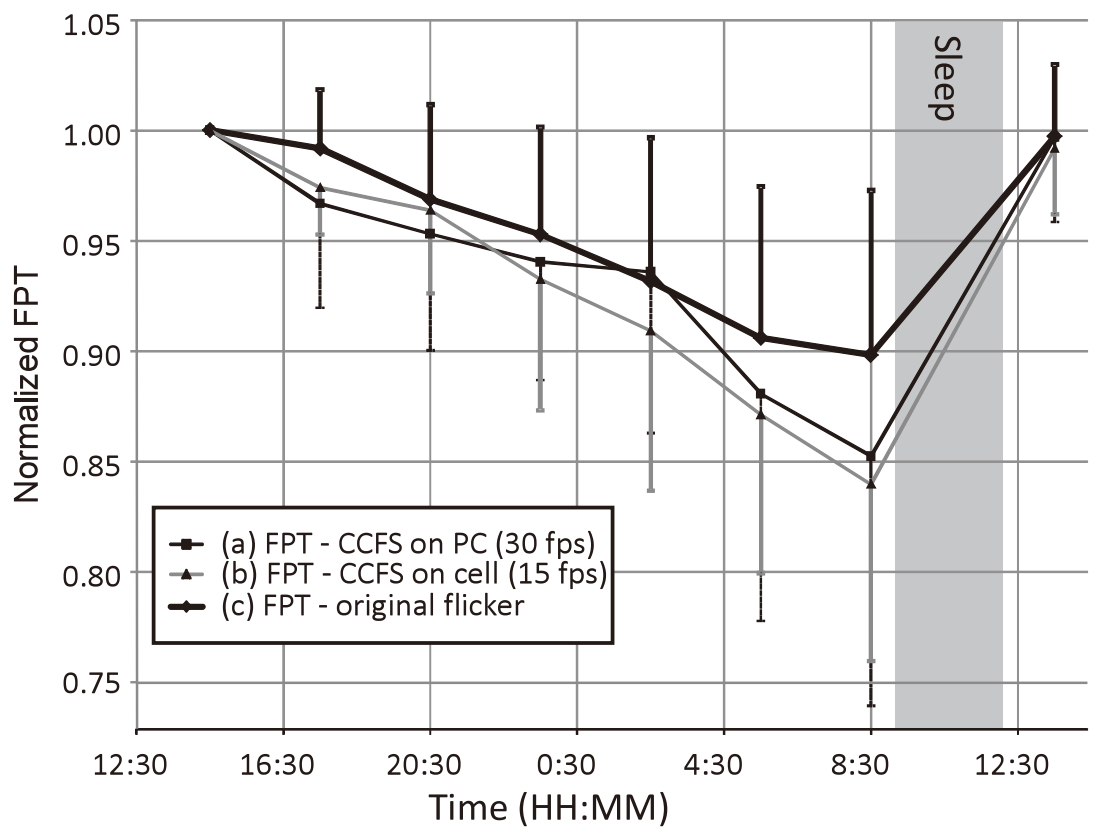

Fig. 3 Temporal changes of the FPT (mean \pm SD) averaged over all subjects during overnight office work setting measured by (a) CCFS implemented as PC software, (b) CCFS implemented on standard cellphone application, and ( $\mathrm{c}$ ) the original flicker test.

Figure 3 shows the temporal changes in FPT during overnight office work averaged over all subjects. Paired ttest with Bonferroni correction for multiple comparison was performed at each time point to detect any significant difference between the two CCFS measurements and the $\mathrm{CFF}$ measured by the original flicker test. No significant difference was found between the FPT measured by two CCFS settings and the original flicker test.

Results of a correlation analysis (Fig. 4) on the FPT obtained from all subjects showed that FPT measured by CCFS tests on a PC correlated significantly with that by the original flicker tests $\left(R^{2}=0.764, p<0.0001\right)$, where $R$ 


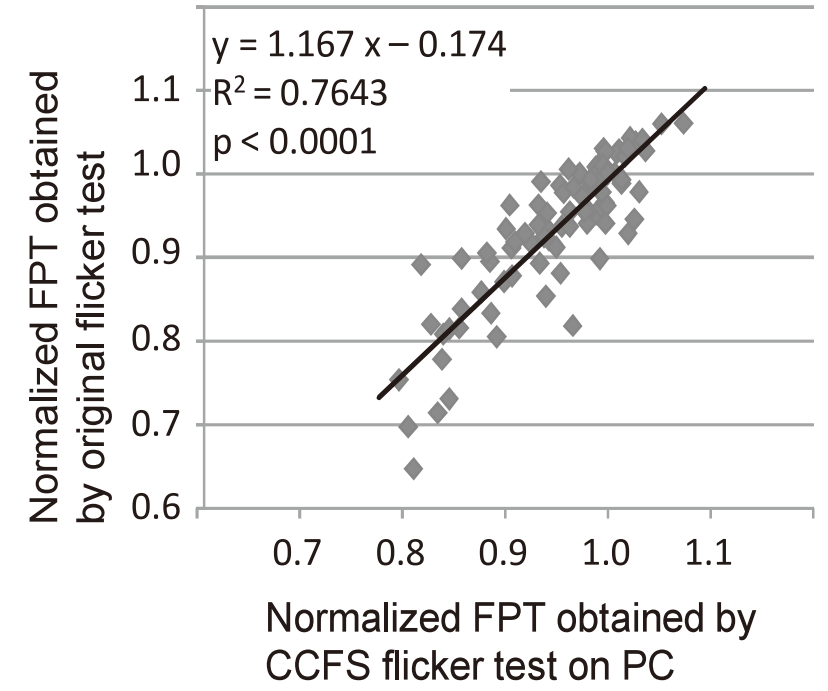

Fig. 4 Results of correlation analysis between FPT obtained by the CCFS flicker test implemented on a PC and FPT obtained by the original flicker test, both normalized by the values at the first measurements for each subject.

is Pearson product-moment correlation coefficient $\left(\mathrm{R}^{2}\right.$ is a coefficient of determination) indicating goodness-of-fit of the linear relationship between FPTs obtained by CCFS tests and the original flicker test, and $\mathrm{p}$ is the result of $\mathrm{t}$ statistics against the null-hypothesis that there is no correlation between the FPTs. A significant correlation was also observed between FPT measured by CCFS test on a cell-phone and that obtained from the original flicker tests $\left(\mathrm{R}^{2}=0.878, \mathrm{p}<0.0001\right)$.

There was a significant correlation between FPTs measured by CCFS tests and the subjective rating of mental fatigue evaluated by VAS $\left(R^{2}=0.472, p<0.0001\right.$ for CCFS on a PC; and $\mathrm{R}^{2}=0.598, \mathrm{p}<0.0001$ for CCFS on a cell-phone). The correlation coefficients between FPTs measured with CCFS and subjective rating were not lower than that measured by the original flicker test $\left(R^{2}=\right.$ $0.442, \mathrm{p}<0.0001$ ).

Results of FPT measurements using FC-FPT parallel the results obtained from the original flicker test during the overnight fatigue measurement sessions (Fig. 5). The time required to complete the FC-FPT test was significantly shorter than that required for the original flicker test (Fig. 6).

\section{Discussion}

We observed a significant correlation between FPTs obtained using CCFS and FPT by the original flicker stimuli. Results of correlation analyses also indicate that the FPTs measured by CCFS correlate significantly with subjective rating of mental fatigue, and that the correlation coefficients obtained using CCFS are even higher than that obtained from the original flicker test. These results indicate that the proposed CCFS could be used to evaluate mental fatigue as measured by the changes in contrast threshold of the flickering sensation. In other

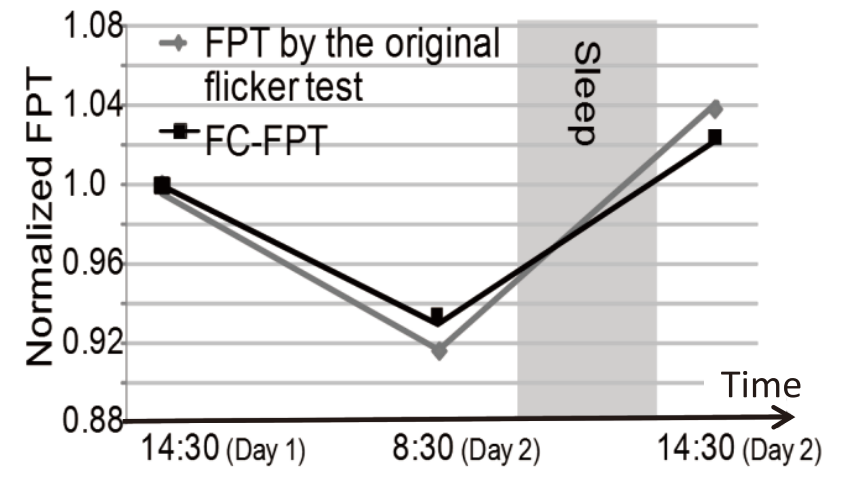

Fig. 5 Comparison between FPTs measured by the original flicker test (gray) and by the FC-FPT test implemented on a PC (black) in a typical subject during overnight fatigue measurement session.

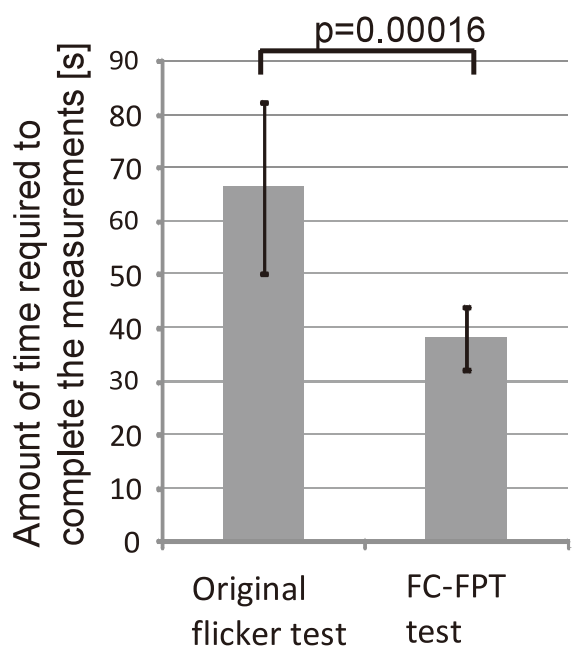

Fig. 6 Time required $($ mean $\pm S D)$ to complete the original flicker test and the proposed FC-FPT test implemented on a PC in a typical subject. Student's t-test was used to determine the significance.

words, CCFS could replace measurement of the temporal frequency threshold used in the original flicker test.

Both CCFS and the original flicker, which measures temporal frequency threshold, appear capable of tracking recovery from mental fatigue imposed by an overnight workload after a brief period of sleep (less than three hours). Moreover, results also indicate that CCFS is able to detect temporary change in mental fatigue after physical exercise. Since FPT reflects the degree of arousal [6], the transient increase in FPT after exercise might represent a temporary increase in arousal level due to increased cardiovascular activity after physical training.

Comparison of the time required to complete the FCFPT test and the original flicker test is shown in Fig. 6. The result indicates that the FC-FPT test is not only effective for controlling arbitrary behaviors of subjects responding to mental fatigue evaluation by FPT measurement, but also significantly reduces the time required to complete the test itself. 


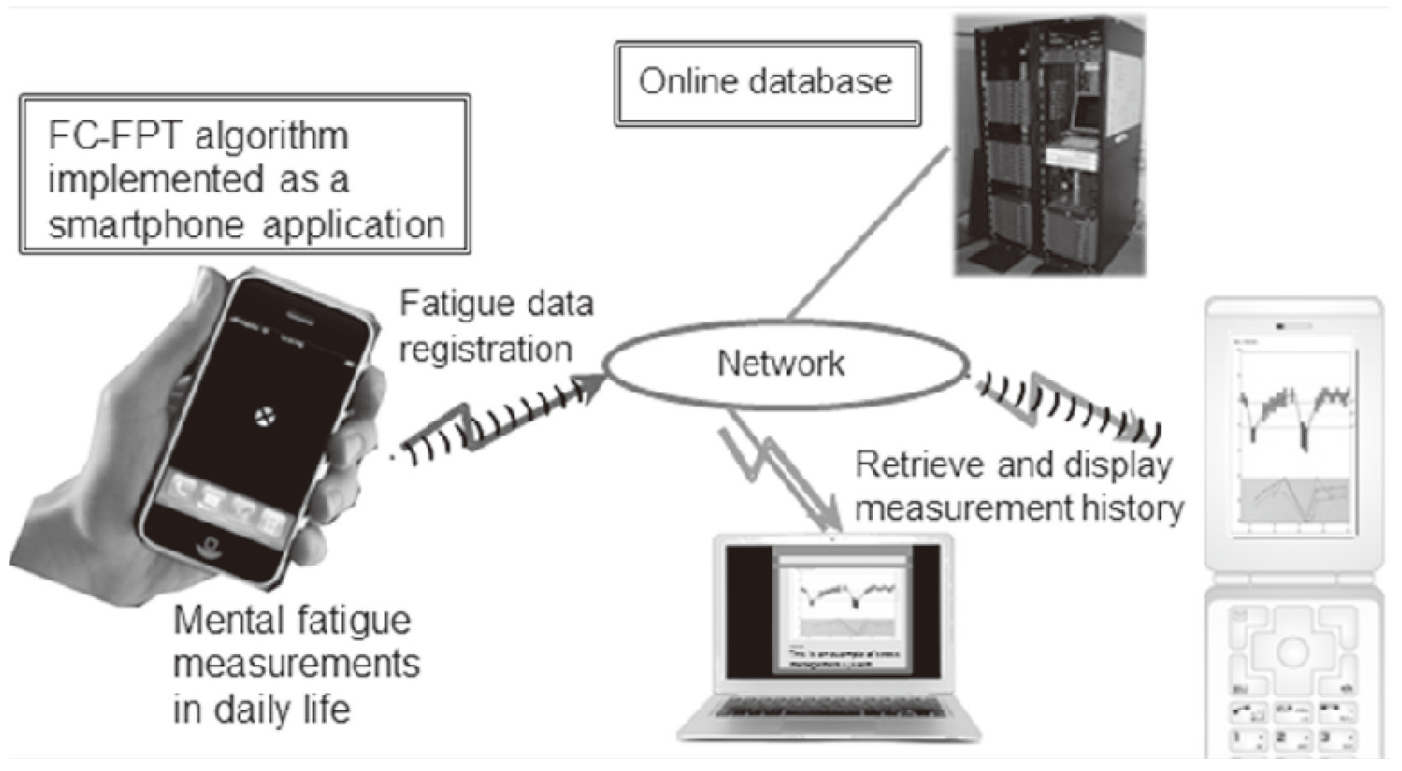

Fig. 7 Proposed prototype of the mental fatigue monitoring system to be used in daily life. Mental fatigue data measured by the FC-FPT algorithm implemented as a smartphone application are automatically stored in the online database that can be retrieved as needed.

The original idea for the development of FC-FPT test using CCFS aimed at allowing the general public a means of conducting quantitative mental fatigue measurements in situations such as the workplace. We have developed application programs to assess changes in mental fatigue over time by measuring flicker perception threshold with CCFS and FC-FPT detection algorithms that run on widely available consumer mobile devices. To facilitate autonomous management of the measured data, we created an internet-based data server. Mental fatigue data measured on mobile devices are uploaded to the database, thus allowing users to objectively track the development of mental fatigue over time, as needed (Fig. 7).

\section{Conclusion}

We developed a new method to control the subjective flickering sensation by changing the contrast of the visual stimuli instead of changing the temporal frequency, and a new paradigm in which forced-choice task performance is used to determine FPT to eliminate arbitrariness in subject responses. The proposed techniques were implemented as application software, which enables persons to measure mental fatigue quantitatively using consumer mobile devices such as smart phones and PCs.

\section{Acknowledgement}

This work was supported in part by the research grants from Mitsui Sumitomo Insurance Welfare Foundation, Suzuki Foundation, and National Institute of Advanced Industrial Science and Technology (AIST).

\section{References}

1. Simonson E, Enzer N: Measurement of fusion frequency of flicker as a test for fatigue of the central nervous system. Indust Hyg Toxicol. 23, pp. 83-89, 1941.

2. Karwowski W: International Encyclopedia of Ergonomics and Human Factors, 2nd Edition, CRC Press, 2006.

3. Hecht S, Verrijp CD: The influence of intensity, color and retinal location on the fusion frequency of intermittent illumination. Proc Natl Acad Sci USA. 19, pp. 522-535, 1993.

4. Hecht S, Verrijp CD: Intermittent stimulation by light: IV. A theoretical interpretation of the quantitative data of flicker. J General Physiol. 17, pp. 269-282, 1993.

5. Grant S, Aitchison T, Henderson E, Christie J, Zare S, McMurray J, Dargie H: A comparison of the reproducibility and the sensitivity to change of visual analogue scales, Borg scales, and Likert scales in normal subjects during submaximal exercise. Chest. 116, pp. 1208-1217, 1999.

6. Tomkiewicz RL, Cohen W: The effects of changes in arousal level on critical flicker fusion frequency and figural reversal tasks. Psychophysiol. 6, pp. 421-428, 1970. 


\section{Sunao IwaKI}

Sunao Iwaki received his B.E. and M.E. degree from Kyushu University in 1990 and 1992, respectively. From 1992 to 1995, he was a research scientist at Hitachi Ltd. He received his Ph.D. degree from University of Tokyo in 1998. Since 1998, he is a research

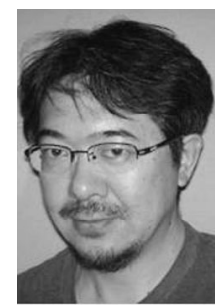
scientist at Electrotechnical Laboratory, National Institute of Advanced Industrial Science and Technology (AIST), where he is working on development of the multimodal neuroimaging techniques and their application to investigate human cognitive functions. He appointed group leader at Human Technology Research Institute, AIST, and is a visiting professor of Osaka University and University of Tsukuba. From 2002 to 2004, he worked at Massachusetts General Hospital/Harvard Medical School as a research fellow. Dr. IwAKI is a member of IEEE BME society, Society for Neuroscience and Organization for Human Brain Mapping.

\section{Nobuyoshi HaRAda}

Nobuyoshi HARAda received his M. Environmental Science degree from Hokkaido University in 1989. From 1991 to 1992, he received French Government Scholarship and studied at University Paris 11. He received his $\mathrm{Ph} . \mathrm{D}$. degree of Environmental

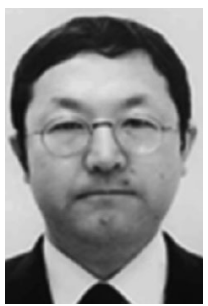
Science from Hokkaido University in 1996. From 1997 to 2000, he was a Domestic Research Fellow of Japan Science and Technology Agency (JST), and from 2000 to 2001, he was a researcher of CREST of JST, and from 2001 to 2003, he was a researcher of New Energy and Industrial Technology Development Organization, at National Institute of Advanced Industrial Science and Technology (AIST), where he studied on effect informational factor in environment to provoke sense of monotony for human. He established Flicker Health Management Corp., and take up a post of president of the company in 2010. Dr. Harada is a member of Society for Neuroscience, Japanese society of Hygiene, and Japanese society of Occupational Health. 